OPEN ACCESS

Edited by:

Mohamed Hijri,

Université de Montréal, Canada

Reviewed by:

Leszek Karliński,

Institute of Dendrology (PAN), Poland

Petr Kohout

Institute of Botany (ASCR), Czechia

*Correspondence:

Yoriko Sugiyama

sugiyama_yoriko@icloud.com

Specialty section:

This article was submitted to Fungi and Their Interactions,

a section of the journal

Frontiers in Microbiology

Received: 21 August 2020

Accepted: 08 February 2021

Published: 18 March 2021

Citation:

Sugiyama $Y$ and Sato $H$ (2021)

The Limited Establishment of Native

Ectomycorrhizal Fungi in Exotic

Eucalyptus spp. Stands in Japan.

Front. Microbiol. 12:597442.

doi: 10.3389/fmicb.2021.597442

\section{The Limited Establishment of Native Ectomycorrhizal Fungi in Exotic Eucalyptus spp. Stands in Japan}

\author{
Yoriko Sugiyama* and Hirotoshi Sato \\ Graduate School of Human and Environmental Studies, Kyoto University, Kyoto, Japan
}

Host specificity may potentially limit the distribution expansion of ectomycorrhizal (ECM) fungi into areas where their original host plants are absent. To test this hypothesis, we investigated whether populations of native ECM fungi may establish in stands of exotic host trees, namely those of the Eucalyptus species, in Japan. ECM fungal communities associated with eucalyptus and surrounding native host species (Pinus thunbergii and Fagaceae spp.) were investigated at two sites; one site in which eucalyptus and native trees were growing in isolation, and a second site in which these species were mixed. To identify fungal taxa, the nuclear ribosomal internal transcribed spacer region 1 was sequenced for the ECM fungi from the root tips and clustered into operational taxonomic units (OTUs). To confirm whether the retrieved OTUs were native to Japan, they were queried against the entire database of the National Center for Biotechnology Information, UNITE, and GlobalFungi, whereby sampling locations and associated hosts were obtained from sequences with $\geq 97 \%$ similarity. Eucalyptus trees were associated with seven and 12 ECM fungal OTUs, including putatively exotic OTUs in isolated and mixed sites, respectively. Among the 36 and 63 native ECM fungal OTUs detected from native hosts at isolated and mixed sites, only one OTU was shared with eucalyptus at the respective sites. This means that most native ECM fungi in Japan may be incapable of forming an association with exotic Eucalyptus spp. Notably, even ECM fungi associated with both Pinus and Quercus were not detected from eucalyptus, suggesting that hostfungus incompatibility is determined not only by host phylogenetic relatedness but also by host biogeographic affinities. Our findings show that the incompatibility with eucalyptus as well as dispersal limitation may prevent the distribution expansion of native ECM fungi in Japan into the distribution ranges of Eucalyptus spp., where the original hosts are absent.

Keywords: ectomycorrhiza, distribution, eucalyptus, exotic host, host compatibility

\section{INTRODUCTION}

Ectomycorrhizal (ECM) fungi form mutualistic associations with a variety of angiosperm and gymnosperm plant species (Smith and Read, 2008). Species belonging to approximately 40 angiosperm families, including Fagaceae, Betulaceae, Dipterocarpaceae, and Myrtaceae, are known to form ECM. Among the gymnosperms, the Pinaceae family is wellknown as an ECM host lineage. It is estimated that a total of 8,500 flowering plant species are capable of forming associations with ECM fungi (Brundrett and Tedersoo, 2018). Nevertheless, due to host specificity, each ECM fungal species forms mutualistic 
associations with a limited range of host lineages within these ECM tree species (Molina et al., 1992; Molina and Horton, 2015). To date, host specificity in ECM fungi has been inferred in various studies (Molina and Trappe, 1994; Sato et al., 2007; Yamada et al., 2010).

Host specificity is a factor that is able to impact the distribution of ECM fungi. For example, Sato et al. (2012) estimated the potential distributions of ECM fungi collected from Yakushima Island, Japan with using the records in GenBank database, and assessed how the distribution ranges of ECM fungi are determined. The aforementioned study demonstrated that most ECM fungi could only be distributed in regions with ECM plant taxa that are similar to those on Yakushima Island, implying that the distribution ranges of ECM fungi are restricted by host specificity rather than by climate preference and dispersal limitations. Since the global distribution of ECM fungi has been rarely investigated (Sato et al., 2012; Tedersoo et al., 2014; GarcíaGuzmán et al., 2017; Bazzicalupo et al., 2018), further studies are required to elucidate the influence of host specificity on the distribution ranges of ECM fungi.

If host specificity is a major factor determining the distribution ranges of ECM fungi, ECM fungi are expected to be incompatible with ECM tree species distributed outside their distribution ranges. To examine this hypothesis, it would be informative to investigate whether ECM fungi can establish associations with host species that have allopatric ranges of distribution; for instance, exotic plant species. Thus far, many ECM trees, such as Pinus spp., Eucalyptus spp., and Acacia spp., have been planted in regions beyond their natural ranges for various purposes (Richardson and Rejmánek, 2011). In these plantations, the richness of associated indigenous ECM fungi has been found to be low (Díez, 2005; Chen et al., 2007; Tedersoo et al., 2007; Nuñez et al., 2009; Dickie et al., 2010; Pennington et al., 2011). Based on this previous evidence, it can be hypothesized that few indigenous ECM fungi can infect exotic tree species due to incompatibility.

However, there is uncertainty as to what extent native ECM fungi are able to infect exotic host trees when the original host plants are absent. Previous studies are generally conducted in forests where native and exotic tree species grow together to a greater or lesser extent. Although some native ECM fungi have been reported to infect exotic host species in such mixed-stand forests (Bahram et al., 2013; Rudawska et al., 2018; SantolamazzaCarbone et al., 2019), caution should be exercised in interpreting these results, as the host ranges of ECM fungi could be broadened in areas where multiple host plants are growing together, owing to infection via common mycelial networks (Smith et al., 1995; Suz et al., 2017). If this hypothesis is true, it is unclear whether ECM fungi associated with exotic hosts in mixed forests are able to establish an association with novel host plant species in areas where their original host plants are absent. To test whether host specificity greatly influences the distribution of ECM fungi, it is necessary to investigate ECM fungal communities in areas populated exclusively by exotic host tree species.

In addition, to assess the aforementioned hypothesis, it would also be vital to compare the associated ECM fungi of exotic trees with those of surrounding native tree species. Some previous studies conducted in pure exotic plant forests have revealed a low diversity of native ECM fungi associated with exotic hosts (Chen et al., 2007; Pennington et al., 2011). However, it remains unclear whether the observed low diversity is due to the incompatibility between native fungi and exotic host species or to the particular characteristics of the study sites (Tedersoo et al., 2007). Thus, to gain a more specific insight into the effect of host specificity, comparisons of the ECM fungal communities associated with neighboring exotic and native hosts are required.

The present study aims to determine whether native ECM fungi is able to colonize exotic host species independently of infection via mycelial networks shared with native host species. To this end, we selected Eucalyptus spp. as the exotic hosts and examined whether the ECM fungi associated with native hosts were shared with eucalyptus trees in two plantation sites in Japan. Whilst trees in the Eucalyptus genus are naturally distributed in Australasia (Hermsen et al., 2012), they have never been part of the native flora of Japan, even in prehistoric times (Hermsen et al., 2012). At the main study site (Kobe Oji Zoo), eucalyptus trees are spatially isolated from other ECM hosts. As such, it is unlikely that infection occurs through mycelial networks shared between native and exotic host species. The barcode sequences of ECM fungi from the root tips were determined and subjected to the basic local alignment search tool (BLAST). The global distributions and host ranges of ECM fungi associated with eucalyptus trees and/or native host species were determined based on annotations of retrieved sequences. A similar survey was carried out at a site where eucalyptus trees were growing amongst native host species (the Botanical Gardens of Osaka City University). The hypothesis tested in this study is that most native ECM fungi are unable to establish populations in eucalyptus plantations, particularly when they are spatially isolated from native species.

\section{MATERIALS AND METHODS}

\section{Sampling Site}

Sampling was conducted at two sites, namely, Kobe Oji Zoo (Kobe, Hyogo, Japan; hereafter Oji Zoo: $34^{\circ} 42^{\prime} 39^{\prime \prime} \mathrm{N}$, $\left.135^{\circ} 12^{\prime} 54^{\prime \prime} \mathrm{E}\right)$ and the Botanical Gardens of Osaka City University (Katano, Osaka, Japan; hereafter Botanical Gardens: $\quad 34^{\circ} 45^{\prime} 54^{\prime \prime} \mathrm{N}, \quad 135^{\circ} 40^{\prime} 51^{\prime \prime} \mathrm{E}$ ) (Table 1 and Supplementary Figure 1). Oji Zoo, which covers an area of $80,618 \mathrm{~m}^{2}$, is located in a residential area, and contains seven species of eucalyptus species planted between 5 and 30 years ago, which are maintained as food for koalas. These eucalyptus species were brought to Japan in the form of seeds from Australia, and were introduced to the zoo as seedlings, having initially been raised in an external nursery. Within the zoo, the trees were planted in several distinct locations (Supplementary Figure 1), at each of which, one to five species have been planted together. Along with these exotics, there are also native Japanese ECM tree species, namely, four Quercus spp. (Q. glauca, Q. phillyraeoides, Q. myrsinifolia, and Q. acutissima) and Pinus thunbergii. These native ECM tree species grow away from the eucalyptus trees (Supplementary Figure 1), and thus there is little likelihood of native ECM fungi infecting eucalyptus trees via common 
TABLE 1 | Description of the sampling sites.

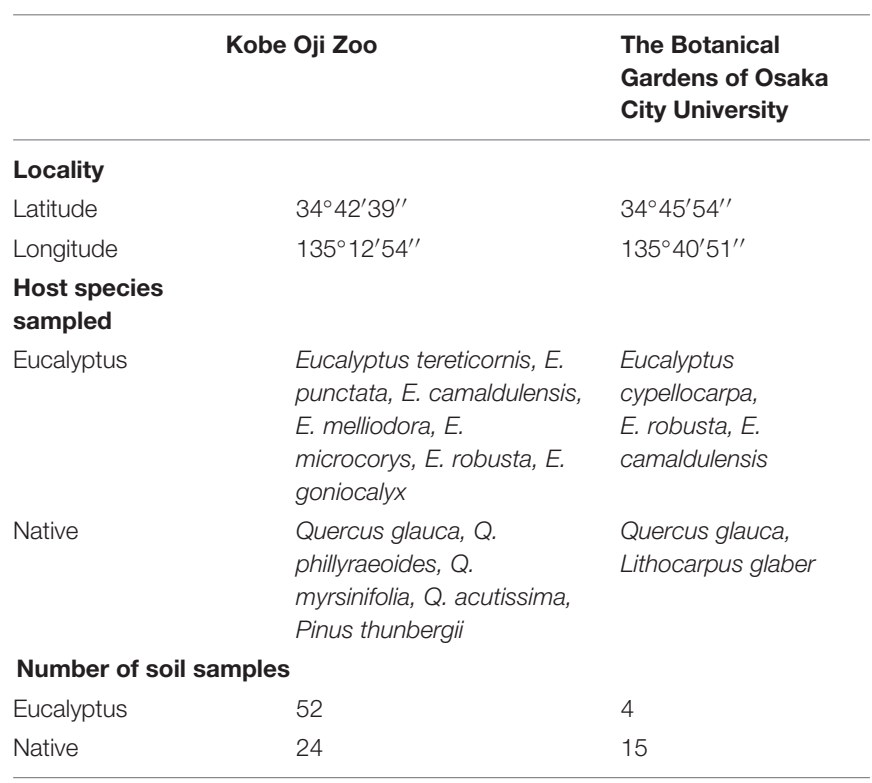

mycelial networks shared with native ECM tree species. In addition to these ECM tree species, several non-ECM tree species (including Cerasus $\times$ yedoensis and Cinnamomum camphora) also grow within the zoo premises.

The Botanical Gardens of Osaka City University extend over an area of 255,300 $\mathrm{m}^{2}$ (including buildings within the gardens). The garden consists of several vegetation areas, among which Australia area is included. In an Australian area, 14 eucalyptus trees were introduced from Tennoji garden (Tennoji, Osaka, Japan) in 2006, and less than 10 now remain. No records are available to indicate the growth stage of the eucalyptus trees when initially introduced to Japan. Although there are no other exotic ECM host species growing within the vicinity of these eucalyptus individuals, some native ECM hosts (Q. glauca and Lithocarpus glaber) do grow nearby or mixed with eucalyptus. Outside the Australia area, Fagaceae species are found commonly throughout the gardens.

Given the small number of resident eucalyptus trees in the Botanical Gardens, greater emphasis was placed on data obtained from the Oji Zoo.

\section{Sampling and Sample Processing}

At each site, soil samples $(10 \times 10 \times 5 \mathrm{~cm})$ containing fragments of the root system of the target host species were collected from beneath the target host species. When the collected soil sample only included a few fine roots, an additional root sample was collected from around the sampling points to obtain more root tips that were enough to check mycorrhizal colonization. In Oji Zoo, whether the obtained roots definitely belonged to the target species was not confirmed because trees belonging to different ECM host families were not growing close together. All samples were taken $>3 \mathrm{~m}$ apart from one other.

At Oji Zoo, 52 eucalyptus and 24 native ECM tree samples were collected in July and December 2019, respectively. The eucalyptus samples were collected from five discrete sites within the zoo premises, each of which was planted with one to five eucalyptus species. However, as comparisons among eucalyptus species was not the purpose of the present study, we did not differentiate among the different species. Four out of five native tree species growing in the zoo were assessed as native ECM trees (Table 1). The breakdown of the 24 samples is as follows: 3 samples from beneath Q. phillyraeoides individuals, seven from Q. glauca, 12 from Q. myrsinifolia, and two from P. thunbergii.

In the Botanical Gardens, four eucalyptus samples and 15 Fagaceae samples were collected in July 2019. The Botanical Gardens records indicated that the sampled eucalyptus species were E. cypellocarpa, E. camaldulensis, and E. robusta. Among the 15 Fagaceae samples, three were from Q. glauca and one was from $L$. glaber, which was growing in close proximity to the eucalyptus trees. The remaining 11 samples were collected from under Fagaceae trees growing at least $50 \mathrm{~m}$ away from the eucalyptus species. Collected samples were maintained in plastic bags and frozen at $-20^{\circ} \mathrm{C}$ until further use.

In the laboratory, each soil sample was slightly defrosted at room temperature $\left(20-25^{\circ} \mathrm{C}\right)$. All fine roots were washed out with tap water. Soil particles and debris were carefully removed from the roots with forceps, and for samples collected from the Botanical Gardens, the roots were divided into different root systems. This is because these samples may have contained both Fagaceae and eucalyptus roots within the same sample. Then, more than 30 root tips (1-2 $\mathrm{mm}$ in length) per sample were collected with the aid of a Stemi DV4 stereo microscope (Carl Zeiss, Oberkochen, Germany) at $\times 20$ magnification. At this point, all root tips showing evidence of ECM fungal colonization (i.e., changes in morphology or color) were sampled. In addition, root tips lacking these features were randomly sampled. For Oji Zoo samples, root tips contained within the same soil sample were pooled (i.e., resulted in 52 eucalyptus and 24 native host samples). For Botanical Gardens samples, root tips from the same root system were pooled to give a single sample, yielding a total 226 (27 eucalyptus and 199 native hosts) samples, which were stored in $70 \%$ ethanol $(\mathrm{w} / \mathrm{v})$ at $-20^{\circ} \mathrm{C}$ until DNA extraction.

\section{DNA Extraction, Polymerase Chain Reaction Amplification, and Sequencing}

Total DNA was extracted from root tips using DNeasy Plant mini kits (Qiagen, Hilden, Germany) following the manufacturer's protocol. To analyze the fungal communities colonizing the roots, the ribosomal internal transcribed spacer regions (ITS15.8S-ITS2) of the ribosomal DNA were amplified using the ITS1F_KYO2 and ITS4_KYO2 (Toju et al., 2012) primer pair fused with an Illumina sequencing primer and six random bases (N). Polymerase chain reaction (PCR) was conducted using $10 \mu \mathrm{L}$ of a KOD FX Neo (TOYOBO, Osaka, Japan) buffer system, containing $1 \times$ PCR buffer, $0.4 \mathrm{mM}$ deoxynucleoside triphosphates, $3 \mathrm{nmol}$ each of the forward and reverse primers, 0.2 units of KOD FX Neo polymerase, and $1.0 \mu \mathrm{L}$ of template DNA. The PCR conditions were as follows: an initial denaturation for $2 \mathrm{~min}$ at $94^{\circ} \mathrm{C}$; followed by 40 cycles of $10 \mathrm{~s}$ at $98^{\circ} \mathrm{C} ; 30 \mathrm{~s}$ at $60^{\circ} \mathrm{C} ; 30 \mathrm{~s}$ at $68^{\circ} \mathrm{C}$; and a final extension for $5 \mathrm{~min}$ at $68^{\circ} \mathrm{C}$. To subsequently fuse the $8 \mathrm{bp}$ identifier indices 
(Hamady et al., 2008) and the MiSeqP5/P7 adapter to the initial PCR amplicons, we conducted an additional PCR using the same PCR mixture and conditions as the initial PCR. The only change to the protocol was that the number of cycles had been reduced to 12. The resultant PCR amplicons were pooled and purified using AMPure XP (Beckman Coulter, Brea, CA, United States). The purified library was then excised using E-Gel SizeSelect (Thermo Fisher Scientific, Waltham, MA, United States). Among the 52 eucalyptus and 24 native samples from Oji Zoo, products had been successfully amplified from 39 and 23 samples, respectively. Among the 27 eucalyptus and 199 native samples collected from the Botanical Gardens, products were amplified from 21 and 178 samples, respectively.

For samples collected from the Botanical Gardens, host tree species identification was conducted simultaneously with fungi, based on the sequence of the ribulose-1, 5-bisphosphate carboxylase/oxygenase large subunit $(r b c L)$ region. To amplify the $r b c L$ region, we used the newly developed primer pair: $5^{\prime}$ TACTGCAGGTACATGYGAA $-3^{\prime}$ as the forward primer, and $5^{\prime}$-ATATGCCAAACGTGAATACC- $3^{\prime}$ as the reverse primer. The PCR mixture and program for the first and second PCRs were the same as those used to amplify the fungal DNA. The exception to this was that the annealing temperature was $55^{\circ} \mathrm{C}$ in the first round of PCR. The amplicon libraries were sequenced via $2 \times 250$-bp paired-end sequencing on the MiSeq platform (Illumina, San Diego, CA, United States) using MISEQ v.2 Reagent Nano Kits, following manufacturer's instructions.

\section{Bioinformatics}

MiSeq sequencing yielded 219 984, 433 496, and 638791 reads for fungi from the Oji Zoo, Botanical Gardens, and plants of the Botanical Gardens, respectively. The retrieved reads were processed using CLAIDENT version 0.2.2018.05.29 (Tanabe and Toju, 2013). Reads were de-multiplexed using the "clsplitseq" command, and the resulting reads were deposited in the Sequence Read Archive of the DNA Data Bank of Japan (accession number: DRA010081). Using the "clfilterseq" command, we trimmed lowquality 3 ' tails, and low-quality reads were filtered based on a minimum quality value of 30 . For fungal reads, the resulting forward and reverse reads were merged using the "clconcatpair" command with the option mode $=$ NON. This mode assumes that there is no overlap between forward and reverse reads, enabling us to concatenate reads in the following order: reverse reads, artificial sequence (ACGTACGTACGTACGT), and forward reads. For plant reads, forward and reverse reads were merged using the option mode $=$ OVL. This mode assumes that there is an overlap between these reads. From the resulting merged reads, potentially noisy and chimeric sequences were eliminated using the "clcleanseqv" command. Remaining reads were clustered into operational taxonomic units (OTUs) with similarity thresholds of 0.97 and 1.00 for fungal ITS and plant $r b c L$, respectively, using the "clclassseqv" command. For OTU clustering of the fungal ITS reads, the artificial sequences were not taken into account. Potentially chimeric OTUs were eliminated using UCHIME (Edgar et al., 2011) without any references.

After removing artificial sequences, OTUs were divided into forward (ITS1) and reverse (ITS2) sequences. The taxonomic assignment of these regions was conducted separately using CLAIDENT. Among the taxonomic assignment results for ITS1 and ITS2, the lower level taxonomic assignment was accepted. In this procedure, OTUs with identifications showing clear differences between ITS1 and ITS2 (e.g., contradiction of taxonomic assignment at the phylum level), were regarded as chimeric and discarded. In addition, in a sample $\times$ OTU matrix, cells with a read number of one were converted to zero to reduce the effects of tag jumps and contamination. After taxonomic assignment with CLAIDENT, we referred to the UNITE database (Nilsson et al., 2019) to determine the taxonomies sequences that could not be assigned to any families or genera using CLAIDENT. Supplementary Table 1 presents the results of the CLAIDENT, UNITE search, and the number of reads for OTUs. The functional groups of the obtained OTUs were inferred using FUNGuild (Nguyen et al., 2016) based on their taxonomy (Supplementary Table 1). Further, for OTUs that were assigned to several functional groups, including ECM (e.g., those identified as Ectomycorrhizal-Fungal Parasite), we referred to reviews by Tedersoo et al. (2010) and Tedersoo and Smith (2013) to verify whether the genus or family of the OTU is included in the list. The resulting ECM fungal OTUs (ECM OTUs) were used in further analyses (see Supplementary Table 1). For the reads of plant samples obtained from the Botanical Gardens, in order to eliminate potentially contaminated or error reads, OTUs with a read number that was less than $10 \%$ of the total read number were eliminated from each sample. The families of the remaining OTUs were then recorded to distinguish whether the host species was a Eucalyptus or a native Fagaceae species. All remaining OTUs were assigned to either Eucalyptus or Fagaceae. As one sample contained both Fagaceae and Myrtaceae OTUs, it was eliminated from further analyses.

\section{Data Analysis}

To compare the ECM fungal colonization rate between the native and eucalyptus host species, Fisher's exact test was conducted in $\mathrm{R}$ version 3.6.0 ( $\mathrm{R}$ Core Team, 2018). In this analysis, the colonization rate was assessed based on the soil sample, and calculated by dividing the number of sequenced soil samples by the number of soil samples from which ECM fungi were detected.

Sample-based OTU accumulation (rarefaction) curves were depicted separately for the ECM fungal communities associated with eucalyptus and native hosts using the "iNEXT" package in R with 1,000 replications. Rarefaction curves for native host species were constructed for two datasets; including and excluding P. thunbergii samples. This was done as phylogenetically distant hosts often harbor distinct ECM fungal communities (Ishida et al., 2007; Matsuoka et al., 2020), which may affect ECM fungal OTU richness. As the number of samples obtained from the Botanical Gardens was relatively small, these statistical analyses were conducted only on samples collected from Oji Zoo.

To identify the potential origins and host range of detected ECM fungal OTUs, we examined the geographic distribution and host range of each OTU. To achieve this, 100 BLASThit sequences were downloaded from the NCBI database, and a p-distance matrix between the focal OTU and retrieved sequences was calculated using MEGA 7 (Kumar et al., 2016). 
It was assumed that sequences with $\geq 97 \%$ similarity in terms of $\mathrm{p}$-distance were derived from the same species. The sampling locations and associated hosts of these sequences were retrieved from the annotation information within the NCBI database. The original publication was consulted in cases where the retrieved country and/or host were not specified in the annotation. The same analyses were conducted on the UNITE and GlobalFungi (Větrovský et al., 2020) databases. In the UNITE search, only sequences without GenBank accession numbers were downloaded. In these evaluations, only sequences retrieved from roots or sporocarps were included, and sequences from soil were removed to exclude the possibility that focal fungus spores had been dispersed at the sampling location without the fungus actually being established.

\section{RESULTS}

\section{The ECM Fungal Community at Oji Zoo}

After filtering and cleaning, there were remaining 52,705 reads from the Oji Zoo samples; these were clustered into 456 OTUs, of which 47 were assigned to ECM fungal taxa (Supplementary Table 1). ECM fungal OTUs were detected in all 23 native host tree samples (i.e., Quercus and Pinus samples), and only in 10 of 39 eucalyptus samples. The rate of fungal infection significantly differed between native host and eucalyptus samples (Fisher's exact test, $p<0.001$ ). Among the 41 OTUs retrieved from native host samples, 10 were from P. thunbergii, 38 were from Quercus spp., and seven were shared between Pinus and Quercus host species. In contrast, only seven ECM fungal OTUs were retrieved from eucalyptus species. One OTU was shared between native and eucalyptus samples (Figure 1), detected in P. thunbergii and Quercus spp. The rarefaction curve for eucalyptus samples almost reached an asymptote, in contrast to that of the native host species, irrespective of whether $P$. thunbergii samples had been included. This indicates the presence of more ECM fungal OTUs associated with native hosts (Supplementary Figure 2).

Taxonomically, among the 41 ECM fungal OTUs retrieved from native host samples, 11 belonged to Ascomycota and 30 belonged to Basidiomycota. These 41 OTUs were classified into 10 families. Although Thelephoraceae was the most OTU-rich family associated with native host species (17 OTUs, 41.5\% of the total number of ECM fungal OTUs), Thelephoraceae OTUs did not dominate in eucalyptus root samples. The seven OTUs retrieved from the eucalyptus samples were identified as Cenococcum geophilum (3), Laccaria sp. (1), Tomentella sp. (1), and Scleroderma albidum (2). Of these, the OTU shared with the native host species was C. geophilum.

\section{Host Plants and ECM Fungal Community in the Botanical Gardens}

All root systems from the four soil samples collected from around the eucalyptus trees belonged to the eucalyptus species, whilst those obtained from the 15 soil samples collected from around native Fagaceae plants belonged to the Fagaceae species. The exception to this was a single sample that contained both

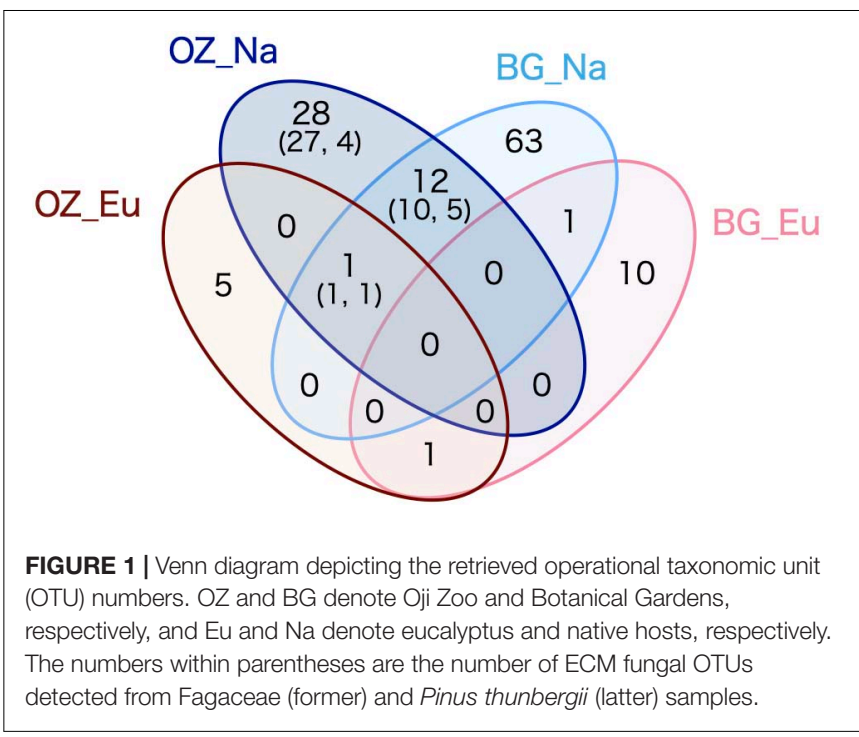

eucalyptus and Fagaceae OTUs, and thus was not used in subsequent analyses. Ectomycorrhizal fungal OTUs were detected in all samples collected from the vicinities of native Fagaceae and eucalyptus trees.

The 247114 reads obtained for fungal ITS were clustered into 678 OTUs, of which 86 were assigned to ECM fungal taxa. Seventy-five ECM fungal OTUs were retrieved from 15 Fagaceae samples, classified into 14 families, among which Thelephoraceae (20 OTUs, 26.7\%) and Russulaceae (17 OTUs, $22.7 \%$ ) were the most OTU-rich families. A comparatively smaller number of ECM fungal OTUs (12) were obtained from four eucalyptus samples, one of which was also detected in native host samples (Figure 1). The 12 ECM fungal OTUs retrieved from the eucalyptus samples were identified as Laccaria spp. (4), Thelephoraceae (3), Tomentella spp., Tuber spp. (2 each), and Sebacina sp. (1). Among these OTUs, one of the Tomentella OTUs was also detected in the native host samples. Furthermore, of the three Laccaria OTUs, one was also detected in the eucalyptus samples obtained from Oji Zoo.

\section{Sampling Localities and Host Species Identified Based on BLAST-Hit Sequences}

Tables 2, 3 and Supplementary Table 2 present the sampling localities and host species of the GenBank, UNITE, and GlobalFungi sequences that showed $\geq 97 \%$ similarity with ECM fungal OTUs detected in the present study (hereafter, BLASThit sequences). Among the 47 and 86 ECM fungal OTUs from Oji Zoo and the Botanical Gardens, eight, and six OTUs, respectively, did not show $\geq 97 \%$ similarity with any reference sequence. Among the ECM fungal OTUs from Oji Zoo and the Botanical Gardens that did show $\geq 97 \%$ similarity with reference sequences, we were unable to verify sampling localities for nine and ten OTUs, respectively. Accordingly, sampling localities and host species were identified for 30 and 70 OTUs, respectively (Supplementary Table 2). 
TABLE 2 | List of ECM fungi detected from eucalyptus, along with the presumed distribution and hosts.

\begin{tabular}{|c|c|c|c|}
\hline $\begin{array}{l}\text { OTU_ID: Species hypothesis ID } \\
\text { taxa }\end{array}$ & Site ${ }^{b}$ & Eucalyptus Forests & Forests of other trees ${ }^{c}$ \\
\hline $\begin{array}{l}\text { OTU_0685: SH1553003.08FU } \\
\text { Laccaria }\end{array}$ & $\begin{array}{l}\mathrm{OZ} \\
\mathrm{BG}\end{array}$ & $\begin{array}{l}\text { Australia, Brazil, Cameroon, Kenya, } \\
\text { Madagascar, Spain, Italy }\end{array}$ & $\begin{array}{l}\text { Argentina (Nothofagus/Carpinus), New Zealand } \\
\text { (Leptospermum/Nohofagus), Australia } \\
\text { (Allocasuarina*/Nothofagus*/Leptospermum*/Acacia*) }\end{array}$ \\
\hline $\begin{array}{l}\text { OTU_0488: SH1544059.08FU } \\
\text { Scleroderma albidum }\end{array}$ & $\mathrm{OZ}$ & & $\begin{array}{l}\text { Argentina (Ilex), Cameroon (Fabaceae), China, } \\
\text { North America }\end{array}$ \\
\hline $\begin{array}{l}\text { OTU_0692: SH1639590.08FU } \\
\text { Cenococcumgeophilum }\end{array}$ & $\mathrm{OZ}$ & & China (Epipactis), USA (Quercus) \\
\hline $\begin{array}{l}\text { OTU_0710: SH1639590.08FU } \\
\text { C.geophilum }\end{array}$ & $\mathbf{O Z}$ & & $\begin{array}{l}\text { New Zealand (Nothofagus), Japan (Pinus), China } \\
\text { (Fagaceae/Pinaceae/Polygonum/Kobresia), } \\
\text { USA (Pyrola/Fagaceae/Betulaceae*/Salix*/Pinaceae*), } \\
\text { Canada (Pinaceae/Quercus/Poplus), Germany (Alnus) }\end{array}$ \\
\hline $\begin{array}{l}\text { OTU_0746: No SH match } \\
\text { Tomentella }\end{array}$ & $\mathrm{OZ}$ & No other record. & \\
\hline $\begin{array}{l}\text { OTU_0764: SH1639590.08FU } \\
\text { C. geophilum }\end{array}$ & $\mathrm{OZ}$ & & Japan (Quercus*/Pinus*/llex*) \\
\hline $\begin{array}{l}\text { OTU_0770: SH1544059.08FU } \\
\text { S. albidum }\end{array}$ & $\mathrm{OZ}$ & $\begin{array}{l}\text { Australia, Brazil, Cameroon, Portugal } \\
\text { China, India, Pakistan, Senegal }\end{array}$ & $\begin{array}{l}\text { Cameroon (Fabaceae*), Cape Verde, Australia, Argentina } \\
\text { (llex), New Caledonia (Acacia), } \\
\text { Estonia (Salix/Populus) }\end{array}$ \\
\hline $\begin{array}{l}\text { OTU_0381: SH1553011.08FU } \\
\text { Laccarialateritia }\end{array}$ & $B G$ & \multicolumn{2}{|c|}{ No information available on the distribution and the host. } \\
\hline $\begin{array}{l}\text { OTU_0416: SH1502458.08FU } \\
\text { C.geophilum }\end{array}$ & $B G$ & \multicolumn{2}{|c|}{ No information available on the distribution and the host. } \\
\hline $\begin{array}{l}\text { OTU_0689: SH1553011.08FU } \\
\text { Laccaria }\end{array}$ & $B G$ & $\begin{array}{l}\text { Australia, Cameroon, } \\
\text { Spain, Sweden, UK }\end{array}$ & $\begin{array}{l}\text { Australia (Nothofagus/Acacia*), New Zealand } \\
\text { (Nothofagus/Leptospermum), Colombia (Acacia) } \\
\text { Germany (Populus), Netherland (Corylus*), Canada }\end{array}$ \\
\hline $\begin{array}{l}\text { OTU_0747: SH1502690.08FU } \\
\text { Tomentella }\end{array}$ & $B G$ & & Germany (Fagus), Japan (Fagaceae*/Pinus*) \\
\hline $\begin{array}{l}\text { OTU_0751: SH1502458.08FU } \\
\text { Tomentella }\end{array}$ & BG & & China (Quercus), Japan (Pinus/Goodyera) \\
\hline $\begin{array}{l}\text { OTU_0753: SH1553011.08FU } \\
\text { Laccaria }\end{array}$ & $B G$ & & Sweden (Salix) \\
\hline $\begin{array}{l}\text { OTU_0759: SH3593769.08FU } \\
\text { Tuber }\end{array}$ & $B G$ & & Italy, USA (Quercus/Malus), Mexico \\
\hline $\begin{array}{l}\text { OTU_0767: SH3593769.08FU } \\
\text { Tuber }\end{array}$ & $B G$ & & No other record. \\
\hline $\begin{array}{l}\text { OTU_0784: No SH match } \\
\text { Sebacina }\end{array}$ & $B G$ & & $\begin{array}{l}\text { France Erica,China (Castanopsis/Pinus/Rhododendron), } \\
\text { USA Ericaceae }\end{array}$ \\
\hline $\begin{array}{l}\text { OTU_0790: SH1503527.08FU } \\
\text { Thelephoraceae }\end{array}$ & $B G$ & & Japan (Fagaceae/Pinus) \\
\hline $\begin{array}{l}\text { OTU_0791: SH1522923.08FU } \\
\text { Thelephoraceae }\end{array}$ & $B G$ & & China (Castanopsis/Pinus/Rhododendron) \\
\hline
\end{tabular}

aspecies Hypothesis ID of best hit sequence in UNITE.

${ }^{b} \mathrm{OZ}$ : Oji Zoo, BG: Botanical Garden. Broad letters indicate that the OTU was also detected in the native host samples at that site.

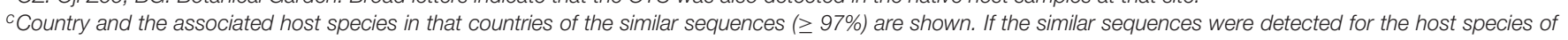
different genera within the same family in a specific country, the family names are indicated instead of listing the genus.

*The associations are uncertain (e.g., samples were collected in a mixed forests).

Among the seven OTUs detected from Oji Zoo eucalyptus samples, we were unable to trace the sampling localities of the BLAST-hit sequences for one OTU (OTU_0746: Tomentella sp.). The sampling localities of the BLAST-hit sequences for OTU_0685 (Laccaria sp.) and OTU_0770 (Scleroderma albidum) were forests in Australia and other countries in the Southern Hemisphere outside the natural distribution of eucalyptus (e.g., Argentina, Cameroon, and New Zealand), along with eucalyptus plantations in other regions (Table 2). However, the BLAST-hit sequences for OTU_0770 included two sequences from Salicaceae forests in Estonia (Table 2). The BLAST-hit sequences of the other Scleroderma albidum OTU (OTU_0488) were from the Northern (North America and China) and Southern (Cameroon and Argentina) Hemispheres. The sampling localities of the three C. geophilum OTUs were largely from Japan, China, and North America, with some from Germany and New Zealand.

Among the 12 OTUs detected from the Botanical Gardens eucalyptus samples, we were unable to identify similar sequences for OTU_0767 (Tuber sp.), and were unable to trace the sampling localities for OTU_0381 (Laccaria lateritia) and OTU_0416 (Thelephoraceae sp.). For one of the three Laccaria OTUs (OTU_0689) not detected in Oji Zoo samples, the 
fungal materials of the BLAST-hit sequences were identified as originating from natural Eucalyptus and Nothofagus forests (e.g., Australia and New Zealand), and forests not containing trees in these genera (e.g., Germany and Netherlands). The BLAST-hit sequences for the remaining seven OTUs were reported to originate only from the natural forests of the Northern Hemisphere (mainly China and Japan), and not from eucalyptus forests.

On the other hand, the BLAST-hit sequences for the OTUs detected from native host samples in the study sites were mainly reported as being from the apparently natural forests of the Northern Hemisphere (Table 3). Hosts for these sequences were reported to be those common to these regions, such as plants of the Fagaceae, Pinaceae, Betulaceae, Salicaceae, Juglandaceae, or Orchidaceae families (Table 3 and Supplementary Table 2). The accession numbers, $\mathrm{p}$-distances to our sequences, sampling locations, associated hosts, and isolation sources for each BLASThit sequence are provided in the Appendix. For OTU_0710 (C. geophilum from an Oji Zoo eucalyptus sample), for which BLAST-hit sequences were obtained from both the Northern (e.g., Japan, China, United States, and Germany) and Southern (New Zealand) Hemisphere, all the BLAST-hit sequences from New Zealand differed by $2.99 \%$ from that of OTU_0710. Accordingly, when the similarity threshold was increased, all the BLAST-hit sequences for OTU_0710 were from the Northern Hemisphere. Apart from this, we found that increasing the similarity threshold did not significantly alter the BLAST results obtained.

\section{DISCUSSION}

In our sites, most of the native ECM fungi seem to be restricted from colonizing eucalyptus trees especially when infection through common mycelial networks is not possible. This appears to be true even for ECM fungi that can associate with both native angiosperms and gymnosperms. These findings support the idea that host-fungus incompatibility is one of the factors that could restrict ECM fungi from expanding their distribution ranges.

The results obtained for Oji Zoo were consistent with the hypothesis that host-fungus incompatibility inhibits the colonization of exotic host species by native ECM fungi. The geographical information obtained from BLAST-hit sequences indicates that the ECM fungal OTUs detected in native host samples could be native to Japan and that they are hardly associated with exotic eucalyptus trees in Oji Zoo (Figure 1 and Tables 2, 3). Given that native hosts were found to harbor many ECM fungal OTUs, it would appear unlikely that a shortage of inocula is the reason the ECM fungi of native hosts did not colonize eucalyptus plants. Moreover, given that we detected a low colonization rate in eucalyptus samples, it seems equally unlikely that competition (Wu et al., 1999; Smith et al., 2018) and/or priority effect (Kennedy et al., 2009; Fukami, 2015) prevent native ECM fungi from colonizing eucalyptus trees. We accordingly consider it more probable that the observed restricted colonization is attributable to hostfungus incompatibility. This assumption would appear to be corroborated by the finding that the ECM fungal species that are putatively identical to those detected only in the native Quercus and Pinus samples are found only in those regions where Fagaceae and Pinaceae are distributed (i.e., Europe, Asia, and North America; Alfaro Reyna et al., 2018). Although it should be highlighted that, in the Southern Hemisphere, there have been comparatively few relevant studies and that the numbers of deposited sequences are currently relatively small (Dickie and Moyersoen, 2008), the results of the present study is consistent with the opinion of Sato et al. (2012) that host-fungus incompatibility limits the distribution range of ECM fungi.

The Oji Zoo results also suggest the possibility that ECM fungi associated with both angiosperm and gymnosperm hosts are incapable of colonizing eucalyptus trees. Some of the native ECM fungal OTUs identified in the present study (e.g., OTU_0704, 0734, and 0787) were detected in both gymnosperms (P. thunbergii) and angiosperms (Quercus spp.), indicating that these OTUs can be categorized as broad host range fungi, according to the conventional definition (Molina et al., 1992). Moreover, among the OTUs that were retrieved from either P. thunbergii or Quercus spp. samples in Oji Zoo, several OTUs are suspected to be associated with both angiosperm and gymnosperm hosts based on an analysis of their BLAST-hit sequences. However, these OTUs were not detected in the root systems of eucalyptus trees in Oji Zoo or elsewhere in the world (Table 3), thereby indicating that even broad host range OTUs may be unable to establish associations with exotic host species. To the best of our knowledge, there have been no previous studies that have tackled the incompatibility between broad host range ECM fungi and exotic hosts. This possibility has, nevertheless, been proposed by Díez (2005), who surveyed the ECM fungal community in a Spanish eucalyptus plantation, although did not assess the host ranges of the native ECM fungi. The findings presented herein provide evidence in support of the hypothesis put forward in Díez (2005), and thereby indicate that host plants that are compatible with ECM fungi are not only determined by phylogenetic relatedness but also by the similarity of their biogeographic background.

Cenococcum geophilum seems to be an exceptional ECM fungal species that is associated with phylogenetically and biogeographically distinct host plants. Although it should be cautioned that C. geophilum is considered to be a species complex (Douhan and Rizzo, 2005; Obase et al., 2016), some OTUs of C. geophilum are shared among continents or host taxa (Ishida et al., 2007; García-Guzmán et al., 2017), indicating that the complex comprises multiple, widespread generalist fungal species. In the present study, we confirmed that a C. geophilum OTU (OTU_0710) had colonized both native and eucalyptus trees in Oji Zoo, under circumstance in which infection through common mycelial networks was unlikely to occur. Furthermore, based on our analysis of BLAST-hit sequences, the same OTU appears to be distributed in a broad swathe of regions, including East Asia, Europe, North America, and Australasia, and can establish mycorrhizal associations with a wide range of host species, including Nothofagus, Betulaceae, Fagaceae, and Pinaceae species. However, given that the 
TABLE 3 | List of ECM fungi detected from native hosts in Oji Zoo, along with presumed distribution and hosts.

\begin{tabular}{|c|c|c|c|c|c|c|c|c|c|c|c|c|}
\hline \multirow[t]{3}{*}{ OTU_ID } & \multirow[t]{3}{*}{ Taxa } & \multirow{3}{*}{$\begin{array}{l}\text { Species } \\
\text { hypothesis ID }\end{array}$} & \multirow{3}{*}{$\begin{array}{l}\text { Host in Oji } \\
\text { Zoob }^{b}\end{array}$} & \multicolumn{7}{|c|}{ Sampling locations $^{c}$} & \multicolumn{2}{|c|}{ Family of presumed hosts } \\
\hline & & & & \multirow[t]{2}{*}{ Europe } & \multicolumn{3}{|c|}{ East Asia } & \multirow[t]{2}{*}{$N^{d}$} & \multirow[t]{2}{*}{$S^{d}$} & \multirow[t]{2}{*}{ Oceania } & & \\
\hline & & & & & Japan & China & Others & & & & & \\
\hline OTU_0151 & Elaphomyces & SH2710213.08FU & Q & & & & & & & & & Fag*, Pin*, Aqu* \\
\hline OTU_0681 & Amanita & SH1611317.08FU & $Q$ & & & & & & & & Pin & Fag, Eri \\
\hline OTU_0686 & $\begin{array}{l}\text { Cenococcum } \\
\text { geophilum }\end{array}$ & SH1522963.08FU & Q & & & & & & & & Pin & Fag, Eri, Orc \\
\hline OTU_0691 & C. geophilum & No SH match & Q & & & & & & & & Pin & Fag, Eri \\
\hline OTU_0695 & C. geophilum & SH1639590.08FU & $Q, P$ & & & & & & & & Pin & $\begin{array}{l}\text { Fag, Bet, Sal, Cyp, } \\
\text { Eri, Not, Euc }\end{array}$ \\
\hline OTU_0710 & C. geophilum & SH1639590.08FU & $E, Q, P$ & & & & & & & & Pin & $\begin{array}{l}\text { Fag, Bet, Sal, Not, } \\
\text { Cyp*, Ros* }\end{array}$ \\
\hline OTU_0717 & Tomentella & SH1524997.08FU & Q & & & & & & & & Pin* & Fag* \\
\hline OTU_0720 & Tomentella & SH1502752.08FU & $Q$ & & & & & & & & Pin* & Fag \\
\hline OTU_0722 & C. geophilum & No SH match & $\mathrm{Q}$ & & & & & & & & & Fag, Orc, Jug \\
\hline OTU_0724 & Inocybe & SH1523975.08FU & $\mathrm{Q}$ & & & & & & & & & Mal, Fag, Bet \\
\hline OTU_0726 & Tomentella & SH1528925.08FU & $\mathrm{Q}$ & & & & & & & & & Eri \\
\hline OTU_0728 & Tomentella & SH1502527.08FU & $\mathrm{Q}$ & & & & & & & & & Fag, Bet \\
\hline OTU_0729 & Tomentella & SH1528925.08FU & Q & & & & & & & & & Fag \\
\hline OTU_0730 & Hymenogaster & SH1563816.08FU & $\mathrm{Q}$ & & & & & & & & Pin* & Fag, Eri, Bet* \\
\hline OTU_0731 & Tomentella & SH1502595.08FU & $\mathrm{Q}$ & & & & & & & & & Fag, Bet \\
\hline OTU_0732 & Tomentella & SH2706606.08FU & Q & & & & & & & & $\operatorname{Pin}^{*}$ & Fag*, Sal* \\
\hline OTU_0734 & Tuber & SH1563428.08FU & $Q, P$ & & & & & & & & Pin & Fag, Sal \\
\hline OTU_0735 & Tomentella & SH1502527.08FU & Q & & & & & & & & No in & ion on the host \\
\hline OTU_0736 & Inocybe & No SH match & $\mathrm{Q}$ & & & & & & & & No in & ion on the host \\
\hline OTU_0743 & C. geophilum & SH1639622.08FU & $\mathrm{Q}$ & & & & & & & & Pin & $\begin{array}{l}\text { Bet, Eri, Fag, Sal, } \\
\text { Jug*, Aqu* }^{*}\end{array}$ \\
\hline OTU_0760 & Tomentella & SH1528513.08FU & $\mathrm{P}$ & & & & & & & & Pin & Bet \\
\hline OTU_0787 & Thelephoraceae & SH1502367.08FU & $Q, P$ & & & & & & & & Pin & Fag, Ros*, Jug* \\
\hline OTU_0789 & Thelephoraceae & No SH match & $\mathrm{Q}$ & & & & & & & & Pin & Fag, Bet \\
\hline OTU_0793 & Hydnaceae & SH2710219.08FU & $Q$ & & & & & & & & Pin & \\
\hline OTU_0962 & Elaphomyces & SH1564355.08FU & $\mathrm{Q}$ & & & & & & & & Pin* & $\begin{array}{l}\text { Fag*, Bet*, Jug*, } \\
\text { Aqu* }^{*}\end{array}$ \\
\hline
\end{tabular}

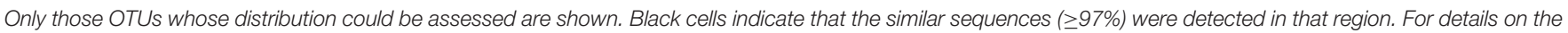

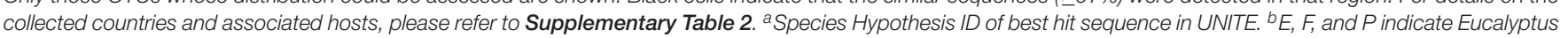
spp., Quercus spp., and P. thunbergii, respectively. ${ }^{c}$ No BLAST-hit sequences were obtained from Russia, Asia outside of East Asia, or Africa. ${ }^{d}$ NA: North Americca,

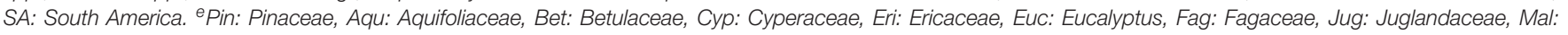
Malvaceae, Orc: Orchidaceae, Ros: Rosaceae, Sal: Salicaceae, Not: Nothofagus. *The association was uncertain (e.g., root samples were collected in a mixed-forests).

OTU_0710 BLAST-hit sequence from Nothofagus forests in New Zealand differed by almost 3\% from the sequence determined in the present study, these findings should be interpreted with caution (Schoch et al., 2012). Nevertheless, collectively, the evidence to date supports the notion that C. geophilum could be distinguished from other ECM fungal species in having an extremely broad host compatibility and distribution range.

Most ECM fungi associated with exotic eucalyptus trees also appeared to be exotic in Japan and originated from those associated with Eucalyptus spp. and other host plants in the Southern Hemisphere, in line with reports from previous studies (Díez, 2005; Pennington et al., 2011). The analysis of BLAST-hit sequences indicated that two OTUs detected from eucalyptus samples in Oji Zoo (OTU_0685,
Laccaria sp. and OTU_0770, Scleroderma albidum) originated from the Southern Hemisphere (Australia, New Zealand, Argentina, or New Caledonia) and eucalyptus plantations. Although eucalyptus species are not native to New Zealand, Argentina, or New Caledonia, the native flora in these countries include ECM host genera that are also native to Australia, such as Nothofagus and Leptospermum. Thus, it is possible that these fungi may be able to establish associations with eucalyptus species and other host species in their original distribution. Indeed, OTU_0685 is presumed to be conspecific to "Trappe14544"; this is an ECM fungus reported to be associated with Nothofagus and Eucalyptus (Wilson et al., 2017). For OTU_0448 (S. albidum), S. albidum is widely known as an introduced species from the Southern Hemisphere, associated with Eucalyptus (Guzmán et al., 2013; 
Sulzbacher et al., 2018). Thus, although none of the BLASThit sequences were from eucalyptus forests, it is possible that the distribution of these BLAST-hit sequences does not reflect the original distribution of OTU_0448. Although the distribution of one OTU (Tomentella sp.) remains unknown, our results indicate that ECM fungi native to Japan are not among the main taxa comprising the ECM fungal community associated with eucalyptus in Oji Zoo. Overall, strong hostfungus incompatibility seems to force exotic eucalyptus trees in Oji Zoo to associate only with ECM fungi growing in their original distribution, although the infection route remains unclear (e.g., long-distance dispersal and/or infection in Japanese eucalyptus nurseries).

The findings of the present study have important implications with respect to our understanding of the biogeography of ECM fungi. Peay et al. (2012) have previously shown that, as the distance from the spore source increases, the number of spores reach and the consequent probability of ECM fungal colonization declines, suggesting that the dispersal limitation is a crucial factor to determine the distribution of ECM fungi. Nevertheless, our results show host-fungus incompatibility between exotic host plant and native ECM fungi growing in the same area (within ca. $250 \mathrm{~m}$ ), suggesting that native ECM fungi detected in our site are not easily established in novel areas where the original host plants are absent, even if a large number of spores reached the region.

Although the number of samples was small, the results obtained for the Botanical Gardens provide clues with respect to determining the conditions under which host-fungus incompatibility might be relaxed. Our findings for both the Botanical Gardens and Oji Zoo were largely comparable, in that we detected a lower ECM fungal OTU richness in eucalyptus samples than in surrounding native host samples, and that only a few OTUs were shared among eucalyptus and native host samples. However, there were also some notable differences in the results obtained from the two sites. In particular, we observed a higher colonization rate in the Botanical Gardens, although statistical significance was not tested. Moreover, based on our BLAST-hit sequence analysis (Table 2), some ECM fungal OTUs in eucalyptus samples collected from the Botanical Gardens (Tomentella spp., Thelephoraceae spp., Sebacina sp., and Laccaria sp.) were seemingly native to Japan. The BLAST-hit sequences also indicated that these OTUs are associated with a range of host species (e.g., Pinaceae, Betulaceae, and Fagaceae) that are distributed in the Northern Hemisphere, specifically in Japan and the surrounding countries (Table 2). We presume that differences in the results obtained for Oji Zoo and the Botanical Gardens might be attributed to the presence/absence of infection via mycelial networks shared between eucalyptus and native hosts, given that eucalyptus and native hosts grow in close proximity in the Botanical Gardens, but not in Oji Zoo. High infection rates in exotic hosts, as well as infection of exotic hosts by native ECM fungi, have also been reported previously in mixed forests composed of both native and exotic hosts (Jairus et al., 2011; Pennington et al., 2011; Moeller et al., 2015; Rudawska et al., 2018; Santolamazza-Carbone et al., 2019). Especially, Santolamazza-Carbone et al. (2019) reported lower infection rates and ECM fungal richness of eucalyptus growing inside the eucalyptus plantations than inside the natural forests, which is in line with our results. Though, in this previous study, indigenous trees were also growing inside the eucalyptus plantations, the low abundance of indigenous trees in these plantations may have made the successive formation of common mycelial networks between native and eucalyptus trees difficult. These results may also support the hypothesis that host-fungus incompatibility could be relaxed by common mycelial networks.

At last, there are some limitations in our study which impose us careful interpretation of the results. First, sampling was conducted at only two sites and there was no replication of either the mixed or isolated eucalyptus stands, which impedes generalizing these results. To confirm generality, it is necessary to conduct similar surveys in different areas and/or with a focus on different exotic host plant species. More empirical studies are also required to confirm whether native ECM fungi are capable of colonizing exotic host trees isolated from native ECM hosts. Second, in Oji Zoo, we collected native and eucalyptus samples in different months. Thus we cannot exclude the possibility that detected difference in ECM fungi between native and exotic hosts may be attributable to seasonal differences. However, the seasonal effect may be small as marked temporal changes in OTU richness and/or community members within the same year have never previously been observed (Koide et al., 2007; Courty et al., 2008; Sugiyama et al., 2020). Further studies addressing these issues would make a substantial contribution to the collective understanding of how host-fungus incompatibility may determine the biogeography of ECM fungi.

\section{CONCLUSION}

This study found that eucalyptus species planted at selected study sites in Japan were associated with exotic ECM fungi that may have the same geographic origins as the exotic eucalyptus trees, while the surrounding native host species formed associations with the native fungi. These results indicate that most ECM fungi detected in the studied sites were incapable of associating with host species originating from regions outside of the potential distribution of the fungi themselves. Our results suggest that ECM fungi observed in the present study do not readily expand their distribution via host shifts to exotic host trees, such as eucalyptus, even if they have dispersed spores into the areas of the Southern Hemisphere. Notably, even ECM fungi associated with both angiosperm (e.g., Quercus spp.) and gymnosperm (e.g., P. thunbergii) hosts at the study sites did not appear to readily infect Eucalyptus species. The findings of the present study suggest the possibility that host-fungus incompatibility is determined not only by the phylogenetic relatedness of host plants but also by the past and present distribution of host plants. Future studies dealing with hosts other than eucalyptus in regions other than our sites would help us understand more fully the relatedness between ECM fungal-host specificity and distribution. 


\section{DATA AVAILABILITY STATEMENT}

The datasets generated in this study can be found in the DDBJ Sequence Read Archive (DRA) under the accession number DRA010081 or BioProject Accession: PRJDB9656.

\section{AUTHOR CONTRIBUTIONS}

YS designed the study, performed the molecular analyses, and wrote the initial draft of the manuscript. YS and HS carried out the field work, analyzed the data, and interpreted the results. HS critically reviewed the manuscript. Both authors contributed to the article and approved the submitted version.

\section{FUNDING}

This study was received partial financial support from a Grantsin-Aid for Scientific Research to HS (20K06796) and Grantsin-Aid for Japan Society for the Promotion of Science Fellows to YS (20J13596).

\section{ACKNOWLEDGMENTS}

We thank Mr. Shosuke Taniguchi and the staff at Kobe Oji Zoo, as well as Dr. Toshihiro Yamada (Faculty of Science, Osaka City University), and the staff at the Botanical Gardens of Osaka City University, for their assistance in fieldwork. We also thank Dr. Hiroki Yamanaka (Center for Biodiversity Science, Ryukoku University) for supporting the laboratory work, particularly the high-throughput sequencing using Illumina MiSeq. We are grateful to Dr. K. S. Rajput (Department of Botany, Faulty of Science, The Maharaja Sayajirao University of Baroda), and Dr. María P. Martín (Real Jardín Botánico, CSIC) for providing information regarding their samples.

\section{REFERENCES}

Alfaro Reyna, T., Retana, J., and Martínez-Vilalta, J. (2018). Is there a substitution of Pinaceae by Fagaceae in temperate forests at the global scale? Glob. Plan. Change 166, 41-47. doi: 10.1016/j.gloplacha.2018.04.001

Bahram, M., Kõljalg, U., Kohout, P., Mirshahvaladi, S., and Tedersoo, L. (2013). Ectomycorrhizal fungi of exotic pine plantations in relation to native host trees in Iran: evidence of host range expansion by local symbionts to distantly related host taxa. Mycorrhiza 23, 11-19. doi: 10.1007/s00572-012-0445-z

Bazzicalupo, A. L., Whitton, J., and Berbee, M. L. (2018). Over the hills, but how far away? Estimates of mushroom geographic range extents. J. Biogeogr. 46, 1547-1557.

Brundrett, M. C., and Tedersoo, L. (2018). Evolutionary history of mycorrhizal symbioses and global host plant diversity. New Phytol. 220, 1108-1115. doi: 10.1111/nph.14976

Chen, Y. L., Liu, S., and Dell, B. (2007). Mycorrhizal status of Eucalyptus plantations in south China and implications for management. Mycorrhiza 17, 527-535. doi: 10.1007/s00572-007-0125-6

Courty, P.-E., Franc, A., Pierrat, J.-C., and Garbaye, J. (2008). Temporal changes in the ectomycorrhizal community in two soil horizons of a temperate Oak Forest. Appl. Environ. Microbiol. 74, 5792-5801. doi: 10.1128/aem.01592-08

\section{SUPPLEMENTARY MATERIAL}

The Supplementary Material for this article can be found online at: https://www.frontiersin.org/articles/10.3389/fmicb. 2021.597442/full\#supplementary-material

Supplementary Figure 1| The locations of the sampling sites and the map of sampling points in Oji Zoo. In the map of Oji Zoo, the areas indicated in pink are eucalyptus stands, and those in blue are native host species. Within the map, the ID of samples taken from the stands is also indicated, which is consistent with the sample ID indicated in Supplementary Table 1.

Supplementary Figure 2 | Sample size-based species accumulation (solid line) and extrapolation (dotted line) curves with 95\% confidence intervals (shaded areas) for the ECM fungal OTU richness of eucalyptus (red) and native (blue) host samples in Oji Zoo. As native host samples, both Fagaceae and Pinus thunbergii samples (i.e., 23 samples) are included in (a) and only Fagaceae samples (i.e., 21 samples) are included in (b).

Supplementary Table 1 | The list of obtained operational taxonomic units (OTUs). OTUs denoted by the "T" in the column, "ECM," are the putative ectomycorrhizal fungal OTUs based on the taxonomy implemented by CLAIDENT. OTUs denoted by the "T" in the column, "ECM_UNITE," are the putative ectomycorrhizal fungal OTUs based on the taxonomy implemented by the CLAIDENT and UNITE searches, and were used for the analyses.

Supplementary Table 2 | The distribution and the associated hosts of the similar sequences. If several countries are listed as collection locations, the country names are listed in parentheses. If similar sequences were detected from host species in different genera within the same family in a specific country, the family names are indicated instead of listing the genera. For samples collected in mixed forests and for which exact hosts were uncertain, the host taxa are indicated in parentheses.*E: Eucalyptus spp., F: Fagaceae spp., P: Pinus thunbergii.

Supplementary Appendix | The results of GenBank, UNITE, and GlobalFungi searches of all ECM fungi retrieved in this study. For each BLAST-hit sequence, the p-distance to the query OTU, the collection site (country), associated host, and sample type are also indicated. Sequences differing by more than $3 \%$ from those detected in this study are denoted by gray letters. Blank cells indicated that we were unable to obtain relevant information. In each seat, the results of the GenBank search are shown in rows without highlights, whilst the results of UNITE and GlobalFungi searches are shown in rows highlighted in yellow and blue, respectively. In UNITE search, sequences that have GenBank accession number or derived from soil are exclude.

Dickie, I. A., Bolstridge, N., Cooper, J. A., and Peltzer, D. A. (2010). Co-invasion by Pinus and its mycorrhizal fungi. New Phytol. 187, 475-484. doi: 10.1111/j. 1469-8137.2010.03277.x

Dickie, I. A., and Moyersoen, B. (2008). Towards a global view of ectomycorrhizal ecology. New Phytol. 180, 263-265. doi: 10.1111/j.1469-8137.2008. 02635.x

Díez, J. (2005). Invasion biology of Australian ectomycorrhizal fungi introduced with eucalypt plantations into the Iberian Peninsula. Biol. Invas. 7, 3-15. doi: 10.1007/1-4020-3870-4_2

Douhan, G. W., and Rizzo, D. M. (2005). Phylogenetic divergence in a local population of the ectomycorrhizal fungus Cenococcum geophilum. New Phytol. 166, 263-271. doi: 10.1111/j.1469-8137.2004. 01305.x

Edgar, R. C., Haas, B. J., Clemente, J. C., Quince, C., and Knight, R. (2011). UCHIME improves sensitivity and speed of chimera detection. Bioinformatics 27, 2194-2200. doi: 10.1093/bioinformatics/ btr381

Fukami, T. (2015). Historical contingency in community assembly: integrating niches, species pools, and priority effects. Annu. Rev. Ecol. Evol. Syst. 46, 1-23. doi: 10.1146/annurev-ecolsys-110411160340 
García-Guzmán, O. M., Garibay-Orijel, R., Hernández, E., Arellano-Torres, E., and Oyama, K. (2017). Word-wide meta-analysis of Quercus forests ectomycorrhizal fungal diversity reveals southwestern Mexico as a hotspot. Mycorrhiza 27, 811-822. doi: 10.1007/s00572-017-0793-9

Guzmán, G., Cortés-Pérez, A., Guzmán-Dávalos, L., Ramírez-Guillén, F., SánchezJácome, M., and del, R. (2013). An emendation of Scleroderma, new records, and review of the known species in Mexico. Rev. Mex. Biodiv. 84, S173-S191.

Hamady, M., Walker, J. J., Harris, J. K., Gold, N. J., and Knight, R. (2008). Error-correcting barcoded primers for pyrosequencing hundreds of samples in multiplex. Nat. Methods 5, 235-237. doi: 10.1038/nmeth.1184

Hermsen, E. J., Gandolfo, M. A., and Zamaloa, M. C. (2012). The fossil record of Eucalyptus in Patagonia. Am. J. Bot. 99, 1356-1374.

Ishida, T. A., Nara, K., and Hogetsu, T. (2007). Host effects on ectomycorrhizal fungal communities: insight from eight host species in mixed conifer-broadleaf forests. New Phytol. 174, 430-440. doi: 10.1111/j.1469-8137.2007.02016.x

Jairus, T., Mpumba, R., Chinoya, S., and Tedersoo, L. (2011). Invasion potential and host shifts of Australian and African ectomycorrhizal fungi in mixed eucalypt plantations. New Phytol. 192, 179-187. doi: 10.1111/j.1469-8137.2011. 03775.x

Kennedy, P. G., Peay, K. G., and Bruns, T. D. (2009). Root tip competition among ectomycorrhizal fungi: are priority effects a rule or an exception? Ecology 90, 2098-2107. doi: 10.1890/08-1291.1

Koide, R. T., Shumway, D. L., Xu, B., and Sharda, J. N. (2007). On temporal partitioning of a community of ectomycorrhizal fungi. New Phytol. 174, 420429. doi: 10.1111/j.1469-8137.2007.02000.x

Kumar, S., Stecher, G., and Tamura, K. (2016). MEGA7: molecular evolutionary genetics analysis version 7.0 for bigger datasets. Mol. Biol. Evol. 33, 1870-1874. doi: 10.1093/molbev/msw054

Matsuoka, S., Sugiyama, Y., Tateno, R., Imamura, S., Kawaguchi, E., and Osono, T. (2020). Evaluation of host effects on ectomycorrhizal fungal community compositions in a forested landscape in northern Japan. R. Soc. Open Sci. 7:191952. doi: 10.1098/rsos.191952

Moeller, H. V., Dickie, I. A., Peltzer, D. A., and Fukami, T. (2015). Mycorrhizal coinvasion and novel interactions depend on neighborhood context. Ecology 96, 2336-2347. doi: 10.1890/14-2361.1

Molina, R., and Horton, T. R. (2015). "Mycorrhiza specificity: its role in the development and function of common mycelial networks," in Mycorrhizal Networks, ed. T. R. Horton (Dordrecht: Springer), 1-39. doi: 10.1007/978-94017-7395-9_1

Molina, R., Massicotte, H., and Trappe, J. M. (1992). "Specificity phenomena in mycorrhizal symbioses: community ecological consequences and practical applications," in Mycorrhizal Functioning, ed. A. Allen (New York, NY: Chapman and Hall), 357-422.

Molina, R., and Trappe, J. M. (1994). Biology of the ectomycorrhizal genus. Rhizopogon. New Phytol. 126, 653-675. doi: 10.1111/j.1469-8137.1994. tb02961.x

Nguyen, N. H., Song, Z., Bates, S. T., Brancoc, S., Tedersood, L., Menke, J., et al. (2016). FUNGuild: an open annotation tool for parsing fungal community datasets by ecological guild. Fung. Ecol. 20, 241-248. doi: 10.1016/j.funeco. 2015.06.006

Nilsson, R. H., Larsson, K.-H., Taylor, A. F. S., Bengtsson-Palme, J., Jeppesen, T. S., Schigel, D., et al. (2019). The UNITE database for molecular identification of fungi: handling dark taxa and parallel taxonomic classifications. Nucleic Acids Res. 47, D259-D264.

Nuñez, M. A., Horton, T. R., and Simberloff, D. (2009). Lack of belowground mutualisms hinders Pinaceae invasions. Ecology 90, 2352-2359. doi: 10.1890/ 08-2139.1

Obase, K., Douhan, G. W., Matsuda, Y., and Smith, M. E. (2016). Revisiting phylogenetic diversity and cryptic species of Cenococcum geophilum sensu lato. Mycorrhiza 26, 529-540. doi: 10.1007/s00572-016-0690-7

Peay, K. G., Schubert, M. G., Nguyen, N. H., and Bruns, T. D. (2012). Measuring ectomycorrhizal fungal dispersal: macroecological patterns driven by microscopic propagules. Mol. Ecol. 21, 4122-4136. doi: 10.1111/j.1365-294x. 2012.05666.x

Pennington, H. G., Bidartondo, M. I., and Barsoum, N. (2011). A few exotic mycorrhizal fungi dominate eucalypts planted in England. Fung. Ecol. 4, 299302. doi: $10.1016 /$ j.funeco.2011.03.002
R Core Team (2018). R: A Language and Environment for Statistical Computing. Vienna: R Foundation for Statistical Computing.

Richardson, D. M., and Rejmánek, M. (2011). Trees and shrubs as invasive alien species - a global review: global review of invasive trees and shrubs. Divers. Distrib. 17, 788-809. doi: 10.1111/j.1472-4642.2011.00782.x

Rudawska, M., Leski, T., Wilgan, R., Karliński, L., Kujawska, M., and Janowski, D. (2018). Mycorrhizal associations of the exotic hickory trees, Carya laciniosa and Carya cordiformis, grown in Kórnik Arboretum in Poland. Mycorrhiza 28, 549-560. doi: 10.1007/s00572-018-0846-8

Santolamazza-Carbone, S., Durán-Otero, M., and Calviño-Cancela, M. (2019). Ectomycorrhizal fungal communities of the alien tree. Sci. Rep. 9:7121.

Sato, H., Tsujino, R., Kurita, K., Yokoyama, K., and Agata, K. (2012). Modelling the global distribution of fungal species: new insights into microbial cosmopolitanism. Mol. Ecol. 21, 5599-5612. doi: 10.1111/mec.12053

Sato, H., Yumoto, T., and Murakami, N. (2007). Cryptic species and host specificity in the ectomycorrhizal genus Strobilomyces (Stromulomycetaceae). Am. J. Bot. 94, 1630-1641. doi: 10.3732/ajb.94.10.1630

Schoch, C. L., Seifert, K. A., Huhndorf, S., Robert, V., Spouge, J. L., Levesque, C. A., et al. (2012). Nuclear ribosomal internal transcribed spacer (ITS) region as a universal DNA barcode marker for Fungi. Proc. Natl. Acad. Sci. U.S.A. 109, 6241-6246.

Smith, G. R., Steidinger, B. S., Bruns, T. D., and Peay, K. G. (2018). Competitioncolonization tradeoffs structure fungal diversity. ISME J. 12, 1758-1767. doi: 10.1038/s41396-018-0086-0

Smith, J. E., Molina, R., and Perry, D. A. (1995). Occurrence of ectoraycorrhizas on ericaceous and coniferous seedlings grown in soils from the Oregon Coast Range. New Phytol. 129, 73-81. doi: 10.1111/j.1469-8137.1995.tb03011.x

Smith, S. E., and Read, D. (2008). Mycorrhizal Symbiosis, 3rd Edn. London: Academic Press.

Sugiyama, Y., Matsuoka, S., and Osono, T. (2020). Two-years of investigation revealed the inconsistency of seasonal dynamics of an ectomycorrhizal fungal community in Japanese cool-temperate forest across years. FEMS Microbiol. Ecol. 96:fiaal18.

Sulzbacher, M. A., Grebenc, T., Bevilacqua, C. B., Steffen, R. B., Coelho, G., Silveira, A. O., et al. (2018). Co-invasion of ectomycorrhizal fungi in the Brazilian Pampa biome. Appl. Soil Ecol. 130, 194-201. doi: 10.1016/j.apsoil.2018. 06.007

Suz, L. M., Kallow, S., Reed, K., Bidartondo, M. I., and Barsoum, N. (2017). Pine mycorrhizal communities in pure and mixed pine-oak forests: abiotic environment trumps neighboring oak host effects. For. Ecol. Manage. 406, 370-380. doi: 10.1016/j.foreco.2017.09.030

Tanabe, A. S., and Toju, H. (2013). Two new computational methods for universal DNA barcoding: a benchmark using barcode sequences of bacteria, archaea, animals, fungi, and land plants. PLoS One 8:e76910. doi: 10.1371/journal.pone. 0076910

Tedersoo, L., Bahram, M., Põlme, S., Kõljalg, U., Yorou, N. S., Wijesundera, R., et al. (2014). Global diversity and geography of soil fungi. Science 346, 1052-1053.

Tedersoo, L., May, T. W., and Smith, M. E. (2010). Ectomycorrhizal lifestyle in fungi: global diversity, distribution, and evolution of phylogenetic lineages. Mycorrhiza 20, 217-263. doi: 10.1007/s00572-009-0274-x

Tedersoo, L., and Smith, M. E. (2013). Lineages of ectomycorrhizal fungi revisited: Foraging strategies and novel lineages revealed by sequences from belowground. Fung. Biol. Rev. 27, 83-99. doi: 10.1016/j.fbr.2013. 09.001

Tedersoo, L., Suvi, T., Beaver, K., and Kõljalg, U. (2007). Ectomycorrhizal fungi of the Seychelles: diversity patterns and host shifts from the native Vateriopsis seychellarum (Dipterocarpaceae) and Intsia bijuga (Caesalpiniaceae) to the introduced Eucalyptus robusta (Myrtaceae), but not Pinus caribea (Pinaceae). New Phytol. 175, 321-333. doi: 10.1111/j.1469-8137.2007.02104.x

Toju, H., Tanabe, A. S., Yamamoto, S., and Sato, H. (2012). High-Coverage ITS primers for the DNA-Based identification of ascomycetes and basidiomycetes in environmental samples. PLoS One 7:e40863. doi: 10.1371/journal.pone. 0040863

Větrovský, T., Morais, D., Kohout, P., Lepinay, C., Algora, C., Awokunle Hollá, S., et al. (2020). GlobalFungi, a global database of fungal occurrences from high-throughput-sequencing metabarcoding studies. Sci. Data 7:228.

Wilson, A. W., Hosaka, K., and Mueller, G. M. (2017). Evolution of ectomycorrhizas as a driver of diversification and biogeographic patterns in 
the model mycorrhizal mushroom genus Laccaria. New Phytol. 213, 1862-1873. doi: $10.1111 / \mathrm{nph} .14270$

Wu, B., Nara, K., and Hogetsu, T. (1999). Competition between ectomycorrhizal fungi colonizing Pinus densiflora. Mycorrhiza 9, 151-159. doi: 10.1007/ s005720050300

Yamada, A., Kobayashi, H., Murata, H., Kalmiş, E., Kalyoncu, F., and Fukuda, M. (2010). In vitro ectomycorrhizal specificity between the Asian red pine Pinus densiflora and Tricholoma matsutake and allied species from worldwide Pinaceae and Fagaceae forests. Mycorrhiza 20, 333-339. doi: 10.1007/s00572009-0286-6
Conflict of Interest: The authors declare that the research was conducted in the absence of any commercial or financial relationships that could be construed as a potential conflict of interest.

Copyright (C) 2021 Sugiyama and Sato. This is an open-access article distributed under the terms of the Creative Commons Attribution License (CC BY). The use, distribution or reproduction in other forums is permitted, provided the original author(s) and the copyright owner(s) are credited and that the original publication in this journal is cited, in accordance with accepted academic practice. No use, distribution or reproduction is permitted which does not comply with these terms. 